\title{
A few days before the end of the 2008 extreme outburst of EX Lupi: accretion shocks and a smothered stellar corona unveiled by XMM-Newton
}

\author{
N. Grosso ${ }^{1}$, K. Hamaguchi ${ }^{2,3}$, J. H. Kastner ${ }^{4}$, M. W. Richmond ${ }^{5}$, and D. A. Weintraub ${ }^{6}$ \\ ${ }^{1}$ Observatoire Astronomique de Strasbourg, Université de Strasbourg, CNRS, UMR 7550, 11 rue de l’Université, \\ 67000 Strasbourg, France \\ e-mail: nicolas.grosso@astro.unistra.fr \\ 2 CRESST and X-ray Astrophysics Laboratory NASA/GSFC, Greenbelt, MD 20771, USA \\ 3 Department of Physics, University of Maryland, Baltimore County, 1000 Hilltop Circle, Baltimore, MD 21250, USA \\ ${ }^{4}$ Center for Imaging Science, Rochester Institute of Technology, 54 Lomb Memorial Drive, Rochester, NY 14623, USA \\ 5 Department of Physics, Rochester Institute of Technology, 84 Lomb Memorial Drive, Rochester, NY 14623, USA \\ ${ }^{6}$ Department of Physics and Astronomy, Vanderbilt University, Nashville, TN 37235, USA
}

Received 10 December 2009 / Accepted 21 June 2010

\section{ABSTRACT}

\begin{abstract}
Context. EX Lup is a pre-main sequence star that exhibits repetitive and irregular optical outbursts driven by an increase in the mass accretion rate in its circumstellar disk. In mid-January 2008, EX Lup, the prototype of the small class of eruptive variables called EXors, began an extreme outburst that lasted seven months.

Aims. We attempt to characterize the X-ray and UV emission of EX Lup during this outburst.

Methods. We observed EX Lup during about $21 \mathrm{~h}$ with XMM-Newton, simultaneously in X-rays and UV, on August 10-11, 2008 a few days before the end of its 2008 outburst - when the optical flux of EX Lup remained about 4 times above its pre-outburst level. Results. We detected EX Lup in X-rays with an observed flux in the $0.2-10 \mathrm{keV}$ energy range of $5.4 \times 10^{-14} \mathrm{erg} \mathrm{s}^{-1} \mathrm{~cm}^{-2}$ during a low-level period. This observed flux increased by a factor of four during a flaring period that lasted about $2 \mathrm{~h}$. The observed spectrum of the low-level period is dominated below $\sim 1.5 \mathrm{keV}$ by emission from a relatively cool plasma $(\sim 4.7 \mathrm{MK})$ that is lightly absorbed $\left(N_{\mathrm{H}} \simeq 3.6 \times 10^{20} \mathrm{~cm}^{-2}\right)$ and above $\sim 1.5 \mathrm{keV}$ by emission from a plasma that is $\sim$ ten times hotter and affected by a photoelectric absorption that is 75 times larger. The intrinsic X-ray luminosity of the relatively cool plasma is $\sim 4 \times 10^{28} \mathrm{erg} \mathrm{s}^{-1}$. The intrinsic X-ray luminosity of EX Lup, $\sim 3.4 \times 10^{29} \mathrm{erg} \mathrm{s}^{-1}$, is hence dominated by emission from the hot plasma. During the X-ray flare, the emission measure and the intrinsic X-ray luminosity of this absorbed plasma component is five times higher than during the low-level period. We detected UV variability on timescales ranging from less than one hour up to about four hours. We show from simulated light curves that the power spectral density of the UV light curve can be modeled with a red-noise spectrum with a power-law index of $1.39 \pm 0.06$. None of the UV events observed on August 10-11, 2008 correlate unambiguously with simultaneous X-ray peaks.

Conclusions. The soft X-ray spectral component is most likely associated with accretion shocks, as opposed to jet activity, given the absence of forbidden emission lines of low-excitation species (e.g., [O I]) in optical spectra of EX Lup obtained during outburst. The hard X-ray spectral component, meanwhile, is most likely associated with a smothered stellar corona. The UV emission is reminiscent of accretion events, such as those already observed with the Optical/UV Monitor from other accreting pre-main sequence stars, and is evidently dominated by emission from accretion hot spots. The large photoelectric absorption of the active stellar corona is most likely due to high-density gas above the corona in accretion funnel flows.
\end{abstract}

Key words. X-rays: stars - stars: individual: EX Lup - stars: pre-main sequence - stars: coronae - stars: activity accretion, accretion disks

\section{Introduction}

Young, low-mass stars that accrete material through their circumstellar disks can exhibit dramatic increases in brightness in the optical. These erupting pre-main sequence (PMS) stars are classified as FUors and EXors, named after the prototypes FU Ori and EX Lup. FUors exhibit a single (observed) major increase in brightness ( $\geq 4-5 \mathrm{mag}$ ), occurring perhaps once per century or only a few times over the PMS lifetime of the star, with a decay timescale of 10-100yr (Herbig 1966, 1977a; Hartmann \& Kenyon 1996). The characteristics of the EXor class are dominated by what is known about the prototype, namely, that the outbursts of EX Lup are repetitive and irregular, occurring perhaps every few decades, with a decay timescale of a few months to a few years (Herbig 1989, 2008). In PMS stars, the typical rates of mass accretion are lower than $\sim 10^{-7} M_{\odot} \mathrm{yr}^{-1}$, but, because of thermal instability, may jump to $\sim 10^{-6}-\sim 10^{-4} M_{\odot} \mathrm{yr}^{-1}$, producing an FUor-type or EXor-type optical outburst (Hartmann \& Kenyon 1996).

Although luminous X-ray emission is characteristic of PMS stars (see reviews of Feigelson \& Montmerle 1999 and Güdel \& Nazé 2009), there exist very few observations of highenergy (UV through X-ray) radiation from FUors or EXors in outburst. An XMM-Newton observation of FU Ori itself shows the cool ( $~ 8 \mathrm{MK})$ and hot ( $\geq 58 \mathrm{MK})$ plasma components usually detected in T Tauri stars (Preibisch et al. 2005), but the absorption toward the hot plasma component is at least 10 times larger than the optical extinction (Skinner et al. 2006). The 
observed X-ray flux is dominated by the hot plasma component, which is evidently due to magnetic activity. However, Skinner et al. (2006) contend that the temperature of the cool component is too high to be caused by accretion shocks. An XMM-Newton observation of the FUor V1735 Cyg detected a hard X-ray spectrum from a hot ( $\geq 58 \mathrm{MK}$ ) plasma (Skinner et al. 2009). In these two FUors, the high temperature of the hot plasma component indicates that the X-ray emission is dominated by magnetic processes. Pre-outburst observations of these FUors in X-rays are not available, hence we are unable to assess the impact of the accretion outburst on their X-ray emission.

In late 2003, V1647 Ori, a young, low-mass star deeply embedded in the L1630 cloud in Orion $(d \sim 400 \mathrm{pc})$ brightened suddenly, illuminating McNeil's nebula. V1647 Ori is the first PMS star to have been observed in X-rays, before, during, and after a major mass-accretion episode (Kastner et al. 2004, 2006b; Grosso et al. 2005; Grosso 2006). Chandra and XMM-Newton observations demonstrate that the sharp increase in X-ray flux post-outburst relative to pre-outburst closely tracked that of the optical/IR brightness increase of V1647 Ori. The X-ray mean flux of V1647 Ori follows the optical/IR decline of the outburst, but enhanced variability on timescales of less than a day was also observed in X-rays. These results appear to be most accurately explained by star-disk magnetic reconnection events that were generated in association with this major mass-accretion episode. V1647 Ori returned to near pre-outburst levels only by late 2005, and was reported to flare again in the optical in late August, 2008 (Itagaki et al. 2008). A new snapshot monitoring with Chandra (Cycle 10; D. A. Weintraub, PI) caught the overall rise/fall shape of this new X-ray outburst (Teets et al. 2010a, in prep.). A Suzaku observation in October 2008 detected strong fluorescent iron line emission that was not present during the 2003-2005 outburst. The structure of the circumstellar gas very close to the stellar core that absorbs and re-emits X-ray emission from the central object may have changed between 2005 and 2008 (Hamaguchi et al. 2010).

The EXor candidate V1118 Ori, a young low-mass star in the Orion Nebula $(d \sim 400 \mathrm{pc})$, displayed a mass accretion outburst from 2005 to 2006 . Follow-up observations of this object detected a moderate enhancement in the X-ray flux that was correlated with the optical/IR flux (Audard et al. 2005, 2010; Lorenzetti et al. 2006).

The Herbig Be/FUor binary star $\mathrm{Z}$ CMa exhibited in 2008-2009 the largest optical outburst reported during the almost 90 years of available observations. The FUor component is responsible for this optical outburst and the X-ray emission of Z CMa. However, no changes in the stellar X-ray properties of the FUor component were seen during this optical outburst (Stelzer et al. 2009). Szeifert et al. (2010) show that the bolometric luminosity of Z CMa remained surprisingly constant during this optical outburst and conclude that the increase in luminosity was caused by a decrease in extinction.

In contrast to the previous results, a Chandra observation was unable to detect the outburst source that illuminates the new nebula associated with IRAS $04376+5413$ in LDN 1415, located at $d \sim 170$ pc (Kastner et al. 2006a; Stecklum 2006; Stecklum et al. 2007).

Given the variety of behavior observed during PMS star outbursts, additional monitoring of PMS star outbursts is essential. We are conducting such a monitoring campaign of potential FUor and/or EXor outbursts, by means of coordinated XMM-Newton and Chandra target of opportunity (ToO) programs. Here, we report on UV/X-ray observations of EX Lup obtained via our XMM-Newton ToO program.
EX Lup is located between the star-forming clouds Lupus 3 and 4 (see review on the Lupus clouds of Comerón 2008), at a distance of $155 \pm 8 \mathrm{pc}$ (Lombardi et al. 2008). It has a normal brightness of $V=13.2 \mathrm{mag}$ (Herbig 2007) and a M0V spectral type (Herbig 1977b). In 1955, the visual observations of Jones helped to infer that EX Lup had experienced a major outburst with a peak magnitude of $V=8.4$ mag (Herbig 1977a). During the 1993-2005 period, eight outbursts not brighter than $V=11$ mag were observed (Herbig et al. 2001; Herbig 2007). Jones (2008a) announced that EX Lup was again in outburst with onset in mid-January 2008. Follow-up visual observations showed that in early February 2008 EX Lup reached a peak $V$-band luminosity of $\sim 8 \mathrm{mag}$, making this last outburst the brightest ever observed from EX Lup (Jones 2008b; Kóspál et al. 2008). On the basis of Spitzer spectroscopy obtained before and during outburst, Ábrahám et al. (2009) claim that heat from this bright outburst transformed, via thermal annealing, amorphous silicate dust in the surface layer of the inner accretion disk of EX Lup into crystalline form (namely, forsterite). They propose that interstellar (amorphous) dust within the protosolar nebula was transformed into crystalline silicate grains present in comets and meteorites during these accretion outbursts.

The 2008 optical outburst of the EXor prototype EX Lup triggered our (anticipated) ToO observations with Chandra (Cycle 9; D. A. Weintraub, PI). This Chandra monitoring of EX Lup with snapshots showed that EX Lup was relatively X-ray bright in March 25, 2008, and had then faded by a factor of three by June 16, 2008, and by another factor of about two by October 2008. The changes in X-ray brightness appear to correlate in a meaningful way with the optical/IR fluxes, which strongly suggests that the level of X-ray production above quiescence for this star is generated by the same physical process that produced the optical eruption, i.e., mass accretion (Teets et al. 2010b, in prep.). The positive detection of EX Lup in X-rays with Chandra allowed us to trigger a long-exposure observation with XMM-Newton (AO-7; Grosso, PI) to perform X-ray CCD spectroscopy and to investigate X-ray and UV variability on a timescale of about one day that we report here. We describe in Sect. 2 the XMM-Newton observation and our reduction of these data. We present our results in Sect. 3, discuss them further in Sect. 4, and offer our conclusions in Sect. 5. We describe in detail our reduction of the Optical/UV Monitor data in Appendix A.

\section{XMM-Newton observation and data reduction}

\subsection{Observing period}

The $78 \mathrm{ks}$ exposure was scheduled at the very beginning of the Summer visibility window of EX Lup (XMM-Newton revolution 1588), on August 10-11, 2008. This observing period is shown in the context of the 2008 extreme outburst of EX Lup in Fig. 1.

For the $V$-band data plotted in Fig. 1, we selected only measurements with error bars from the American Association of Variable Star Observers (AAVSO) ${ }^{1}$ and from the ASAS-3 photometric catalog 2 of the All Sky Automated Survey (ASAS; Pojmanski 2002). We estimated the pre-outburst optical level of EX Lup using the median of $V$ measurements obtained on Sep.-Oct. 2007, just before the conjunction of EX Lup with the Sun. We found that $V \simeq 12.4 \mathrm{mag}$, which is above its normal

\footnotetext{
1 See the AAVSO site at http: //WwW . aavso . org.

2 The ASAS-3 photometric catalog is available at http://www . astrouw . edu.pl/asas/.
} 
N. Grosso et al.: accretion shocks and a smothered stellar corona unveiled by XMM-Newton

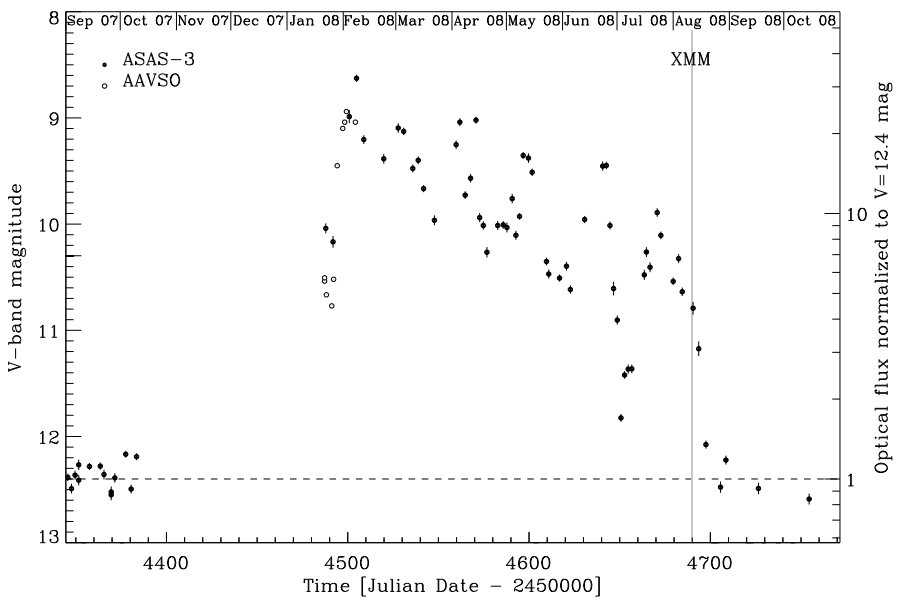

Fig. 1. $V$-band light curve of EX Lup. Filled and empty circles are $V$-band magnitudes of EX Lup from the All Sky Automated Survey (ASAS-3) and the American Association of Variable Star Observers (AAVSO), respectively. The horizontal dashed line is the pre-outburst optical level. The right $y$-axis gives the optical flux in pre-outburst flux units. The grey stripe at JD $\simeq 2454690$ identifies the XMM-Newton observing period in August 2008.

brightness of $V=13.2$ mag (Herbig 2007). EX Lup returned to this pre-outburst optical level on late August, 2008.

Figure 1 shows that our XMM-Newton observation was obtained only a few days before the end of the 2008 outburst, but that EX Lup was clearly still in an elevated state during the observation. Ten hours after the end of the XMM-Newton observation, EX Lup was observed by the ASAS at $V=10.79 \pm$ 0.06 mag. For comparison, this optical level is as bright as the peak of the brightest outburst of the 1993-2005 period that was observed on August, 2002, and 4.4 times above its 2008 preoutburst optical level.

\subsection{Instrumental setup}

The prime instrument was EPIC, with pn (Strüder et al. 2001) and the two MOS (Turner et al. 2001), used in the full frame science mode with the medium optical blocking filter.

The Optical/UV Monitor (OM; Mason et al. 2001) was used in the default fast mode with the $U V M 2$ filter (covering the wavelength band from 205 to $245 \mathrm{~nm}$, with an effective wavelength of $231 \mathrm{~nm}$ ), which allowed us to image a $17^{\prime} \times 17^{\prime}$ field-of-view and obtain for EX Lup fifty UV event lists (1200 s observations separated by gaps of $321.6 \mathrm{~s}$ due to overhead time). Because, during our OM observation, no good guide stars were found, we were unable to use the XMM-Newton Science Analyzing System $(\mathrm{SAS})^{3}$ to correct the UV event lists of EX Lup for spacecraft drifts. We present in Appendix A the method we used to correct the OM fast-mode light curve for spurious features caused by spacecraft drifts.

\subsection{Target identification}

The pipeline data products (PPS) provide correlations of EPIC sources in our observation with archival catalogs. The EPIC source XMMU 160305.5-401825, with a positional uncertainties of 1 .'56 (including a 1 '.50 systematic error), is located only 0.'7 from the coordinate position of 2MASS J160305484018254 (=EX Lup). Therefore, this EPIC source is the X-ray

${ }^{3}$ See

http://xmm.esac.esa.int/external/xmm_data_analysis/. counterpart of EX Lup. The closest neighboring X-ray source detected by XMM-Newton is located at a distance of $2 ! 3$ from the EX Lup source. The Cycle 9 Chandra images show that the closest X-ray source to EX Lup is a faint object located at a distance of $44^{\prime \prime}$. Therefore, the X-ray and UV flux from XMMU 160305.5-401825 are not contaminated by flux from any nearby source.

From the PPS list of OM sources detected in the $17^{\prime} \times 17^{\prime}$ field-of-view, we correlated the positions of OM sources of a signal-to-noise ratio $(S / N)$ greater than 3.5 with the positions of 2MASS sources ${ }^{4}$, which corrects the OM source positions for a small pointing offset $\left(\Delta \alpha=1{ }^{\prime \prime} 6 \pm 0.2\right.$ and $\Delta \delta=-2 . .7 \pm$ 0.2 ), leading to a position uncertainty of 0.3 . We find a bright UV source located only 0.'3 from 2MASS J16030548-4018254. Therefore, this OM source is the UV counterpart of EX Lup.

\subsection{Data reduction}

The data were reprocessed and analyzed using the SAS (version 9.0) following standard procedures ${ }^{5}$. Source plus background X-ray events ${ }^{6}$. were extracted within a circular region centered on EX Lup that had been optimized to maximize the $S / N$ (which gives a $14^{\prime \prime}$ radius for $\mathrm{pn}$ ). The background X-ray events located on the same CCD were extracted using an annular region centered on EX Lup. We applied to the X-ray light curve of each camera both relative corrections (dead time, GTIs, exposure, background counts) and absolute corrections (vignetting, bad pixels, chip gaps, PSF, quantum efficiency) with the SAS task epiclccorr. Therefore, we report the count rates that would have been detected if the X-ray events had been collected with an infinite extraction radius.

All the EPIC spectra presented here were binned to at least 15 counts per spectral bins. Spectral modeling was performed with XSPEC (version 12.5.1; Dorman \& Arnaud 2001). We used $\mathrm{X}$-ray spectra from optically-thin plasmas in collisional ionization equilibrium, which include continuum and emission line output from the Astrophysical Plasma Emission Code ${ }^{7}$ (vapec model in XSPEC). We adopted the abundance pattern of the $X M M-N e w t o n$ Extended Survey of the Taurus molecular cloud (XEST; Güdel et al. 2007a), which we take to be the typical elemental abundances of the coronae of young stars, as measured with grating X-ray spectroscopy ${ }^{8}$. We combined these emission spectra with photoelectric absorption using the XSPEC model wabs, which is based on the photo-ionization cross sections of Morrison \& McCammon (1983) and the solar abundances of Anders \& Ebihara (1982).

\footnotetext{
4 We use the SAS task eposcorr.

5 See SAS threads at http://xmm.esac.esa.int/sas/current/ documentation/threads/.

${ }^{6}$ For PN, we selected only single and double pixel events (i.e., PATTERN in the 0 to 4 range) with FLAG value equal to zero. For MOS, we selected single, double, triple, and quadruple pixel events (PATTERN in the 0 to 12 range), and used the FLAG list \#XMMEA_SM, which is advised for accurate spectral analysis (see the SAS task emchain)

${ }^{7}$ For more informations on APEC, see

http: //hea-www . harvard. edu/APEC.

8 The abundance pattern of the XEST (Güdel et al. 2007a) is: $\mathrm{C}=0.45$, $\mathrm{N}=0.788, \mathrm{O}=0.426, \mathrm{Ne}=0.832, \mathrm{Mg}=0.263, \mathrm{Al}=0.5, \mathrm{Si}=0.309$, $\mathrm{S}=0.417, \mathrm{Ar}=0.55, \mathrm{Ca}=0.195, \mathrm{Fe}=0.195$, and $\mathrm{Ni}=0.195$ (with respect to the solar photospheric abundances of Anders \& Grevesse 1989).
} 


\subsection{Effective exposure}

This observation was affected by bad space weather (Fig. 2). In particular, the last $3 \mathrm{ks}$ of the exposure were strongly affected by highly elevated flaring-background. Therefore, we did not use EPIC data obtained on August 11, 2008 after about 13:00 (i.e., after about $37 \mathrm{~h}$ in Fig. 2).

For the time variability study presented in Sect. 3.1, this $X M M$-Newton observation with an effective exposure of $\sim 74 \mathrm{ks}$ is the longest continuous X-ray (and UV) observation that was obtained during this optical outburst of EX Lup.

For the spectral analysis presented in Sect. 3.2, we suppressed time intervals with flaring background rate above 8.0 pn count $\mathrm{ks}^{-1} \mathrm{arcmin}^{-2}$, which reduces the effective exposure for the pn spectrum to only $\sim 22 \mathrm{ks}$ (see time periods shown in grey in Fig. 2).

\section{Results}

\subsection{X-ray variability}

To limit the background, we concentrated our variability analysis on the energy range from $0.2 \mathrm{keV}$ to $7.3 \mathrm{keV}$. We divided this energy range into soft $(S, 0.2-1.7 \mathrm{keV})$ and hard $(H$, $1.7-7.3 \mathrm{keV})$ bands to allow a hardness ratio study using the formula $H R=(H-S) /(H+S)$. We used $1500 \mathrm{~s}$ for the bin size of the X-ray light curves of each camera to ensure that each time bin has a positive count rate in at least one of the three cameras. We replaced the negative count rates by zero.

Figure 3 shows the background-subtracted X-ray light curves of EX Lup obtained by adding the pn, MOS1, and MOS2 light curves. During the first two-thirds of the observation, EX Lup exhibited a low level of activity, with amplitude variation of about 0.02 EPIC counts $\mathrm{s}^{-1}$ around an average count rate of about 0.03 EPIC counts $\mathrm{s}^{-1}$. Then, just after $31.2 \mathrm{~h}$, as shown in the top panel of Fig. 3, we observed a rapid increase in the count rate that peaked at $\sim 3$ times the average low-level count rate, before decaying to the pre-outburst level at the end of the observation. This last, decay phase, occurring just after $33 \mathrm{~h}$, was strongly affected by the highly elevated background. The source flare-like event was detected mainly in the hard band, and was the only clear variability detected in this energy band. There was also a possible fainter second source flare-like event seen in both the hard and soft X-ray bands around $20.5 \mathrm{~h}$, but this time interval was strongly affected by a large flaring background; therefore, we cannot conclude definitively that EX Lup experienced two flares, though it certainly experienced only one bright flare peaking above 0.07 count s$^{-1}$ during this $74 \mathrm{ks}$ observing window.

The bottom panel of Fig. 3 shows that the hardness ratio is soft during the low-level period, hard during the main period of the source flare lasting about $6.5 \mathrm{ks}$ (see vertical dashed lines in Fig. 3), and returns to softer values during the decay phase and the end of the observation. We conclude that this variability and this hardening is characteristic of an X-ray flare from EX Lup.

For the pn, MOS1, and MOS2 light curves in the soft band, we applied a Kolmogorov-Smirnov (KS) test ${ }^{9}$ to establish whether the variations are consistent with Poisson noise that is associated with a constant source. For each of the three light curves, we found a KS-test probability below 0.001 , which

\footnotetext{
9 We used the ftools task lcstats (xronos subpackage). ftools is available at http://heasarc.nasa.gov/lheasoft/ftools/ ftools_menu.html.
}

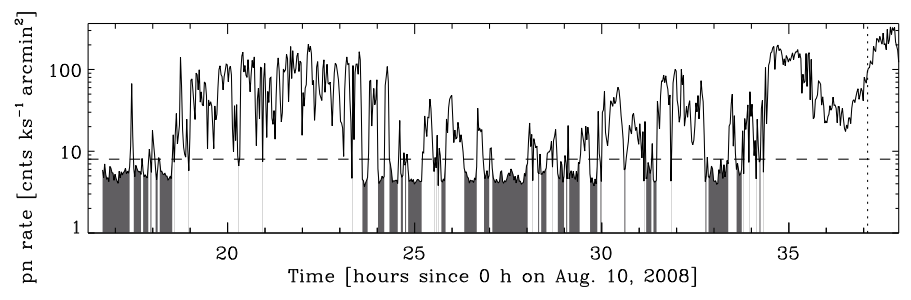

Fig. 2. Flaring-background light curve in the pn detector during our $X M M-N e w t o n$ observation of EX Lup. The line is the background light curve produced by the SAS task epchain. The horizontal dashed line indicates the maximum level of flaring-background for our spectral analysis. The resulting time periods with low flaring-background are shown in grey. The vertical dotted line indicates the end of the useful exposure.

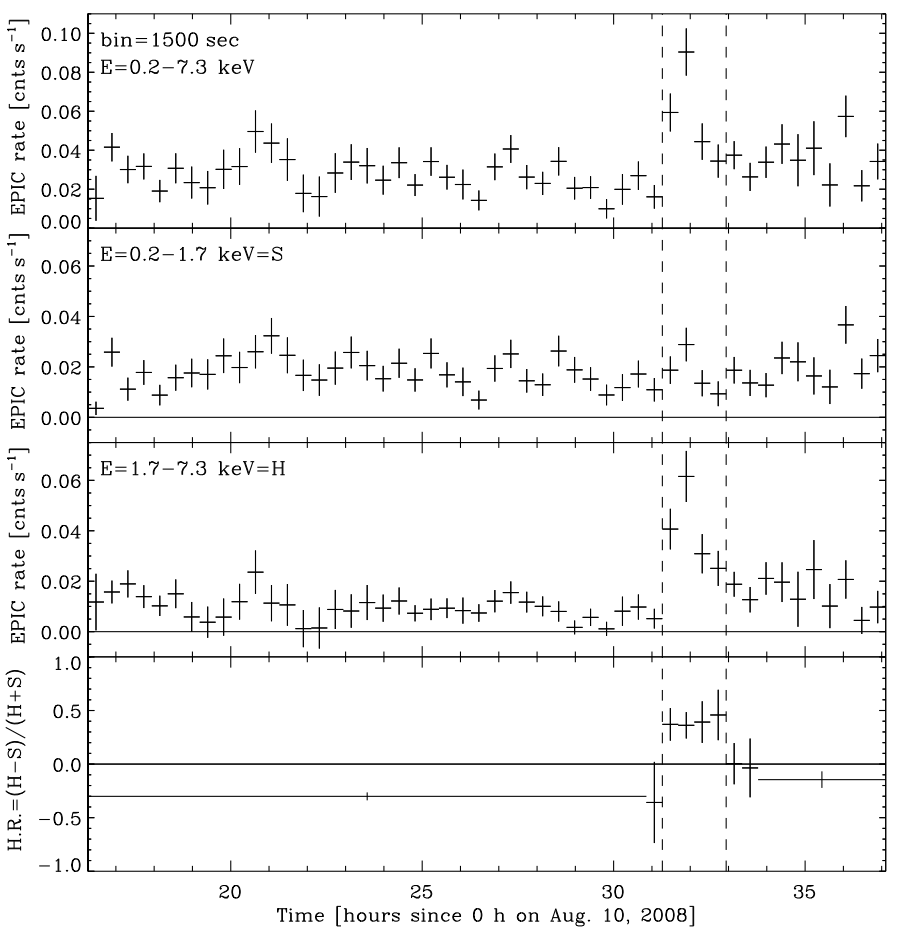

Fig. 3. XMM-Newton/EPIC background-subtracted X-ray light curves of EX Lup. The top panel shows the EPIC (pn+MOS1+MOS2) X-ray light curve of EX Lup in the energy band from 0.2 to $7.3 \mathrm{keV}$. The middle panels show the soft $(S=0.2-1.7 \mathrm{keV})$ and hard $(H=$ $1.7-7.3 \mathrm{keV}$ ) band X-ray light curves. The bin size of the X-ray light curves is $1500 \mathrm{~s}$. The bottom panel shows the variation of the corresponding hardness ratio. The vertical dashed lines bracket the source flaring period.

indicates a reliable level of soft-band variability (e.g., Getman et al. 2005).

\subsection{Time-dependent $X$-ray spectral analysis}

The MOS spectra of EX Lup are very faint hence cannot be used to provide useful constraints from spectral modeling; the spectral modeling results we present were therefore obtained exclusively from the pn spectra. We first performed X-ray modeling of the data from the low-level period of our observation, before the source flare that began at $31.2 \mathrm{~h}$ (see vertical dashed lines in Fig. 3); this low-level period has a low flaring-background exposure time of $18.6 \mathrm{ks}$ (see Fig. 2). The top panel of Fig. 4 shows the spectrum of the low-level period. It is dominated by 
Table 1. Physical parameters of the X-ray emission from EX Lup obtained by spectral fitting of the pn data.

\begin{tabular}{|c|c|c|c|c|c|c|c|c|c|c|c|}
\hline \multirow[b]{2}{*}{ Period $^{a}$} & \multicolumn{3}{|c|}{ Soft spectral component } & \multicolumn{3}{|c|}{ Hard spectral component } & \multirow[b]{2}{*}{$\begin{array}{c}F_{\mathrm{X}}^{b} \\
\left(\operatorname{erg~cm}{ }^{-2} \mathrm{~s}^{-1}\right)\end{array}$} & \multirow{2}{*}{\multicolumn{2}{|c|}{$\begin{array}{c}L_{\mathrm{X}, \mathrm{s}}{ }^{c} \quad L_{\mathrm{X}, \mathrm{h}}{ }^{c} \\
\left(10^{28} \mathrm{erg} \mathrm{s}^{-1}\right)\end{array}$}} & \multirow[b]{2}{*}{$\chi^{2} /$ d.o.f. } & \multirow[b]{2}{*}{$Q^{d}$} \\
\hline & $\begin{array}{c}N_{\mathrm{H}, \mathrm{s}} \\
\left(10^{20} \mathrm{~cm}^{-2}\right)\end{array}$ & $\begin{array}{c}T_{\mathrm{s}} \\
(\mathrm{MK})\end{array}$ & $\begin{array}{c}E M_{\mathrm{s}} \\
\left(10^{51} \mathrm{~cm}^{-3}\right)\end{array}$ & $\begin{array}{c}N_{\mathrm{H}, \mathrm{h}} \\
\left(10^{22} \mathrm{~cm}^{-2}\right)\end{array}$ & $\begin{array}{c}T_{\mathrm{h}} \\
(\mathrm{MK})\end{array}$ & $\begin{array}{c}E M_{\mathrm{h}} \\
\left(10^{51} \mathrm{~cm}^{-3}\right)\end{array}$ & & & & & \\
\hline$L$ & $\begin{array}{c}3.6 \\
\leq 14.8\end{array}$ & $\begin{array}{c}4.7 \\
3.6-7.9\end{array}$ & $\begin{array}{c}4.2 \\
2.9-7.9\end{array}$ & $\begin{array}{c}2.7 \\
0.4-7.3\end{array}$ & $\begin{array}{c}53 \\
\geq 18\end{array}$ & $\begin{array}{c}18.3 \\
7.9-114\end{array}$ & $0.54 \times 10^{-13}$ & 4.3 & 29.2 & $10.38 / 12$ & 0.58 \\
\hline$F$ & $=3.6$ & $\begin{array}{c}4.2 \\
3.0-6.8\end{array}$ & $\begin{array}{c}5.7 \\
3.9-7.5\end{array}$ & $\begin{array}{c}5.2 \\
2.6-11.2\end{array}$ & $\begin{array}{c}61 \\
\geq 19\end{array}$ & $\begin{array}{c}102 \\
51-594\end{array}$ & $2.3 \times 10^{-13}$ & 5.6 & 170 & $4.03 / 8$ & 0.85 \\
\hline
\end{tabular}

Notes. The model used for the photoelectric absorption is wabs. The model used for the continuum and emission lines produced by an optically thin plasma in thermal collisional ionization equilibrium is vapec, using the abundance pattern of the XMM-Newton Extended Survey of the Taurus molecular cloud (XEST; Güdel et al. 2007a). The second line of each period corresponds to the confidence intervals at the $90 \%$ confidence level (i.e., $\Delta \chi^{2}=2.706$ for each parameter of interest). ${ }^{(a)}$ The low-level and the flaring period are labeled $L$ and $F$, respectively. ${ }^{(b)}$ Observed X-ray flux in the $0.2-10 \mathrm{keV}$ energy range. ${ }^{(c)} \mathrm{X}$-ray luminosity corrected for the absorption in the $0.2-10 \mathrm{keV}$ energy range, at a distance of $155 \mathrm{pc}$ (Lombardi et al. 2008). ${ }^{(d)}$ The $Q$-value is the probability that one would observe the chi-square value, or a larger value, if the assumed model is true, and the best-fit model parameters are the true parameter values.
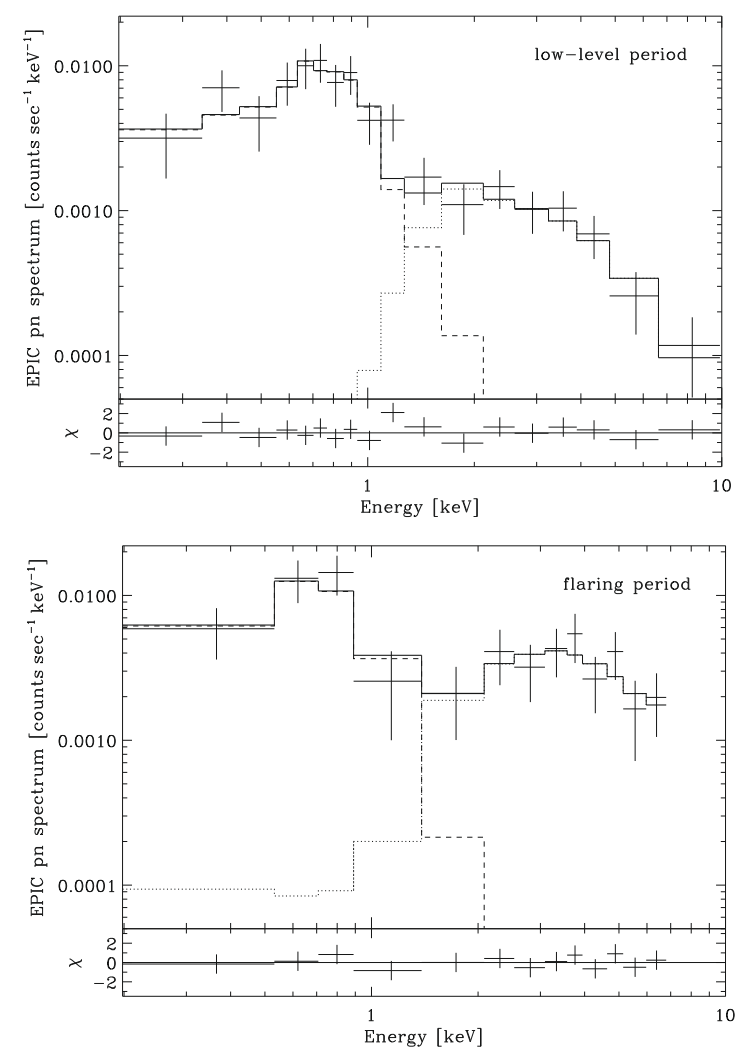

Fig. 4. pn spectra of EX Lup. Top panel: low-level emission. Bottom panel: flaring emission. The continuous, dashed and dotted lines are the best model of the whole spectrum, and the soft and hard components (see Table 1), respectively. The residuals of the fit (in sigma units) are shown in the bottom sections of each panel.

soft emission that peaks around $0.7 \mathrm{keV}$, which suggests that the emission below $\sim 1.5 \mathrm{keV}$ originates in a relatively cool plasma. $\mathrm{X}$-rays were detected at energies as low as $0.2 \mathrm{keV}$, which indicates that the photoelectric absorption is low, and at energies as high as $10 \mathrm{keV}$.

A single-component model consisting of thermal plasma emission combined with a very low photoelectric absorption provided a poor fit ( $\chi^{2}=27.84$ for 15 degrees of freedom) and can be statistically rejected (i.e., the probability that the best-fit model matches the data, $Q=0.023$, is lower than 0.05 ).
When we added a second plasma component to the model of the flux during the low-level period, where both components were affected by the same photoelectric absorption, we obtained a statistically acceptable fit $\left(\chi^{2}=15.86\right.$ for 13 degrees of freedom, corresponding to $Q=0.26)^{10}$. However, the best-fit value for the hot plasma temperature corresponds to the highest temperature available in vapec and the resulting lower limit of 175 MK is too high for a PMS star (e.g., Preibisch et al. 2005). Moreover, the residuals of the fit show a dip in the 1.2-2.0 keV energy range and an excess in the $2.0-5.0 \mathrm{keV}$ energy range, suggesting that the modeling could be improved. We conclude that the spectral shoulder above $\sim 1.5 \mathrm{keV}$ prevents us from obtaining any satisfactory model fit to the full spectrum during the low-level period if the model includes a single value for the absorbing column density for both plasma components.

Hence, to simultaneously fit both the soft and hard spectral components, we introduced a second absorber (hydrogen column densities, $N_{\mathrm{H}, \mathrm{s}}$ and $N_{\mathrm{H}, \mathrm{h}}$, for the soft and hard spectral components, respectively). Procedurally, we first fitted the spectrum at energies below $1.5 \mathrm{keV}$, thereby obtaining best-fit values for $N_{\mathrm{H}, \mathrm{s}}$, the plasma temperature $\left(T_{\mathrm{s}}\right)$, and the emission measure $\left(E M_{\mathrm{s}}\right)$ for the soft spectral component. Fixing these bestfit model parameters, we then fitted the whole spectrum, and obtained best-fit values for model parameters $\left(N_{\mathrm{H}, \mathrm{h}}, T_{\mathrm{h}}, E M_{\mathrm{h}}\right)$ for the hard spectral component. Using these six best-fit parameters as initial conditions, we then fit the whole spectrum, allowing all the parameters vary. With these initial conditions, the model parameters varied only slightly and provided to a good fit (first line of Table 1). Using the F-test, we verified that adding this second absorber increases the goodness-of-fit at the $5 \%$ significance level. The soft spectral component is modeled with lightly absorbed $\left(N_{\mathrm{H}, \mathrm{s}} \simeq 3.6 \times 10^{20} \mathrm{~cm}^{-2}\right)$ emission from a 4.7 MK plasma. The hard spectral component is modeled with emission from a $53 \mathrm{MK}$ plasma affected by much larger absorption $\left(N_{\mathrm{H}, \mathrm{s}} \simeq 2.7 \times 10^{22} \mathrm{~cm}^{-2}\right)$. The X-ray luminosities of the soft and the hard components, after correcting for absorption, are $\sim 4.3 \times 10^{28}$ and $\sim 29.2 \times 10^{28} \mathrm{erg} \mathrm{s}^{-1}$, respectively. Therefore,

10 The best-fit model parameters are : a very low photoelectric absorption of $6.0 \times 10^{20} \mathrm{~cm}^{-2}\left(1.7 \times 10^{20}-21 \times 10^{20} \mathrm{~cm}^{-2}\right.$ at the $90 \%$ confidence level); a relatively cool plasma with a temperature of $3.8 \mathrm{MK}$ $(2.6-6.2 \mathrm{MK})$ and an emission measure of $2.8 \times 10^{51} \mathrm{~cm}^{-3}(1.2 \times$ $\left.10^{51}-15.6 \times 10^{51} \mathrm{~cm}^{-3}\right)$; and a very hot plasma with a temperature of $794 \mathrm{MK}(\geq 175 \mathrm{MK})$ and an emission measure of $8.1 \times 10^{51} \mathrm{~cm}^{-3}$ $\left(5.4 \times 10^{51}-10.1 \times 10^{51} \mathrm{~cm}^{-3}\right)$. 
the intrinsic X-ray emission of EX Lup is dominated by emission from the hotter plasma.

The bottom panel of Fig. 4 shows the spectrum during the flaring period, from 31.2 to $33.0 \mathrm{~h}$ of the whole exposure of $\sim 6.5 \mathrm{ks}$ (Fig. 3). The shape of the spectrum during the flare incorporates both soft and hard spectral components, with some similarity but also significant differences in overall shape from the spectrum obtained during the low-level period. The greatest difference between the flare and low-level spectra is the greater prominence of the hard component during the flare. As for the low-level emission, a model of the flare period spectrum in which both temperature components are subject to the same absorbing column fails. Such a model again cannot fit the data in the 1.2-2.0 and 2.0-5.0 keV energy ranges, and results in an unrealistically high value for the temperature of the hot component. Therefore, we used a model for which both plasma components were affected by different photoelectric absorption when fitting the flare spectrum; however, the statistics of the soft spectral component during the flare are very poor, due to the short flare duration. The weak signal from the soft component during the flare precludes us from constraining $N_{\mathrm{H}, \mathrm{s}}$ from the flare spectrum. We therefore assumed that the absorption of the soft spectral component did not change during the flare and we fixed the value of $N_{\mathrm{H}, \mathrm{s}}$ to that obtained from modeling the low-level spectrum.

Our model fit to the overall flare spectrum inferred for the soft spectral component a plasma temperature and emission measure similar to that of the low-level period (second line of Table 1). For the hard spectral component, we found a higher value for the absorption and the plasma temperature of the hard spectral component during the flare $\left(5.2 \times 10^{22} \mathrm{~cm}^{-2}\right.$ versus $2.7 \times 10^{22} \mathrm{~cm}^{-2}$, and $61 \mathrm{MK}$ versus $53 \mathrm{MK}$, respectively). Given the range of possible values in our confidence interval, however, we cannot conclude with confidence that the absorption and the plasma temperature had increased. We can conclude, though, that the emission measure and the intrinsic X-ray luminosity of the hard spectral component increased by a factor of $\sim$ five during the flare.

After the source flaring period, the source faintness and the brevity of the exposure with low-background level available after 33 h (i.e., $2.5 \mathrm{ks}$, see Fig. 2) did not allow us to obtain a useful spectrum.

\subsection{UV variability}

The UV event lists produced by the OM fast mode were rebinned to a bin size of $120 \mathrm{~s}$ to increase the signal-to-noise ratio. We obtained $10 \mathrm{UV}$ measurements per OM fast-mode exposure, thus, $500 \mathrm{UV}$ measurements for the whole observation. Figure 5 shows this UV light curve and, for comparison, the X-ray light curve. Significant UV variability was detected, with several events lasting from shorter than one hour to as long as about four hours. During these one-to-four hour events, the UV flux increased by ten to twenty percent from the initial count rate before returning to it. The baseline level of the UV light curve was not constant. In the first half of the observation, it followed a downward trend from about 40 counts $\mathrm{s}^{-1}$ to a minimum of about 36 counts $\mathrm{s}^{-1}$, and, then, an upward trend to a maximum of about 45 counts $\mathrm{s}^{-1}$.

None of the multiple UV peaks correlated simultaneously with any of the possible changes in the X-ray count rate. The rise of the X-ray flare occurred about $2 \mathrm{~h}$ after the start of a large UV flare. However, we cannot conclude, definitively, that

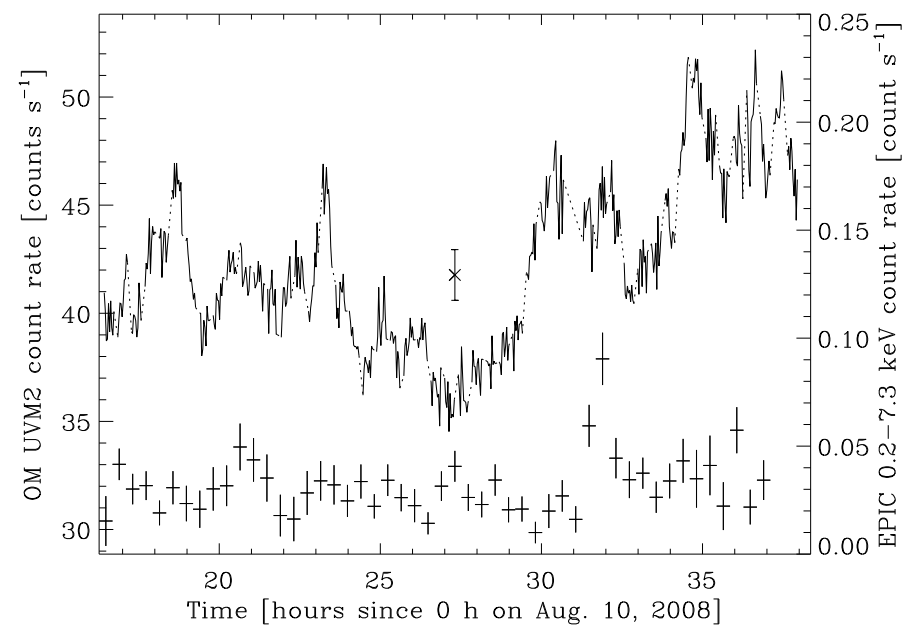

Fig. 5. Simultaneous UV and X-ray light curves of EX Lup. The upper and lower curves are the OM light curve (left $y$-axis) and the EPIC light curve (right $y$-axis), respectively. The UV light curve obtained with the OM fast mode was rebinned to bin size of $120 \mathrm{~s}$. In the figure center, the cross with error bar indicates the median value and median error of the UV photometry. To guide the eye, the dotted lines link consecutive OM exposures. The bin size of the X-ray light curve is $500 \mathrm{~s}$.

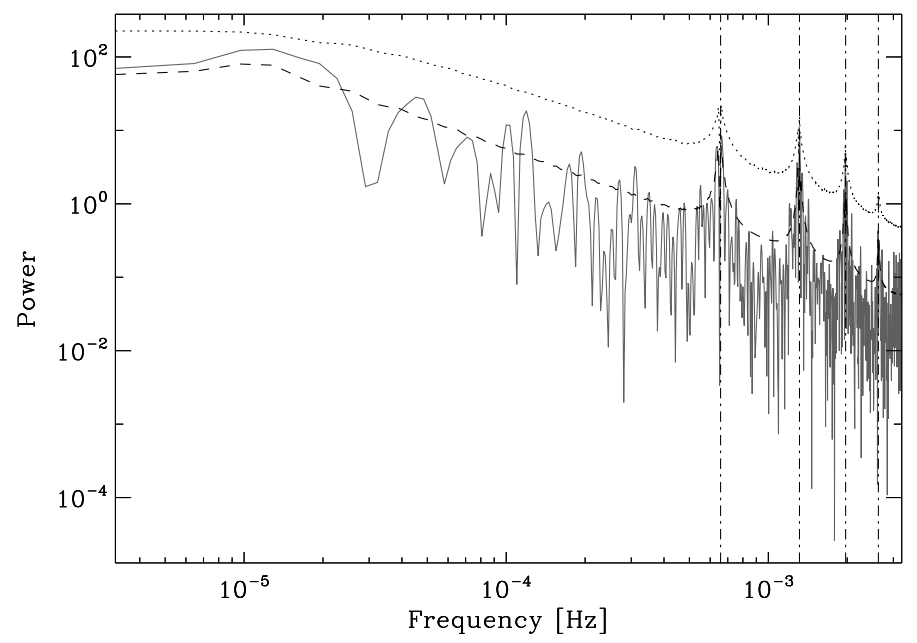

Fig. 6. Lomb normalized periodogram (LNP) of the UV light curve. The grey line is the LNP of the OM light curve. The dashed and dotted lines are the average value and the $3 \sigma$ upper threshold of the LNP of red noise with power-law index of 1.39. The vertical dashed-dotted lines indicate the frequency associated with our observing set-up and harmonic values $(n=2,3,4)$.

absolutely no connection exists between the UV and X-ray flare events.

To search for any periodicity in this UV light curve, we computed the Lomb normalized periodogram (LNP; Lomb 1976; Scargle 1982; Press \& Rybicki 1989), which is well suited to unevenly sampled time series. The LNP revealed that the power of the UV signal versus frequency has a power-law shape (i.e., red noise), in addition to several high frequency peaks (Fig. 6). A least squares $\log -\log$ fitting of the LNP versus frequency inferred a negative slope, $a=-1.26 \pm 0.05$. However, our observing procedure may flatten the LNP at high frequencies (e.g., Uttley et al. 2002), hence the value of the LNP slope is not 
simply the negative of the power-law index $(\alpha)$ of the true power spectral density (PSD), $\operatorname{PSD}(f) \propto f^{-\alpha}$, where $f$ is the frequency (see our simulations below). Nevertheless, this best-fit value of $a$ indicates that $\alpha$ is most likely to be between 1 and 2, which are values of $\alpha$ associated with flicker noise and random-walk noise (Press 1978), respectively. In contrast to the case of Gaussian white noise $(\alpha=0)$, where the significance level of any peak in the LNP can be evaluated analytically (Scargle 1982), we needed here to perform detailed simulations to assess the level of significance of the high frequency peaks.

We assumed first that the true PSD of the intrinsic UV time series is that of red noise that we sampled evenly at the Nyquist frequency of our observation $\left(f_{\text {Nyquist }}=2 / 120 \mathrm{~Hz}\right)$ from $60 \mathrm{~s}$ to 10 times the duration of our observation, and we then used the algorithm of Timmer \& Koenig (1995) to produce the corresponding light curve. We divided this light curve into 10 shorter light curves matching the duration of our observation. Each light curve was then binned in the same way as our UV light curve, and scaled to match the UV mean count rate and standard deviation. The corresponding LNPs were then computed, leading to a sample of $10 \mathrm{LNP}$. We repeated this procedure 10000 times to obtain a large (100000) sample of LNPs. We found that red noise with $\alpha=1.39 \pm 0.06$ reproduces the slope of the LNP of the observed UV light curve. The observed high frequency peaks are below the upper threshold of the $99.7 \%$ confidence level (equivalent to $3 \sigma$ in the Gaussian case) of the random fluctuation of the LNP, and are, hence, not significant. Indeed, our simulated light curves display similar high frequency peaks that we identify as aliasing produced by our observing windows. The lower frequency $\left(f \approx 6.6 \times 10^{-4} \approx 1 / 1522 \mathrm{~Hz}\right)$ matches the frequency associated with the sum of fast mode exposure (1200 s) and exposure gap (321.6 s); the other frequencies are harmonics $(n \times f$, with $n=2,3,4)$.

We conclude that the UV variability can be modeled with red noise $(\alpha=1.39 \pm 0.06)$ that arises from a true variability of the underlying physical process. We detected no significant, periodic signal in the UV light curve.

\section{Discussion}

\subsection{Two-absorber $X$-ray sources}

The X-ray spectrum of EX Lup exhibits two plasma components, one that is cooler with low absorption and one that is hotter with high absorption. Similar soft and hard spectral components with two absorbers have previously been observed in a few low-mass stars and protostars with accretion disks and jets that have been detected in forbidden emission lines (Kastner et al. 2005; Güdel et al. 2005; Güdel et al. 2007b).

The acronym TAX, which stands for Two-Absorber X-ray, was introduced by Güdel et al. (2007b) to classify jet-driving sources that display this peculiar X-ray spectrum. The interpretation of the X-ray spectra of young stellar objects classified as TAX sources is that variable emission from the hotter plasma of the stellar corona is absorbed by circumstellar material, producing the hard spectral component. The non-variable, lightly absorbed soft component, meanwhile, likely arises as a consequence of shocks located at the base of jet-launching zones.

The prototype of the FUor class, FU Ori, shows this kind of unusual X-ray spectrum, but the $\sim 8 \mathrm{MK}$ temperature of the soft component in FU Ori may reflect a coronal magnetic process rather than a shock origin (Skinner et al. 2006).

\subsection{The origin of the hard X-ray component of EX Lup}

The flaring activity of the hard component of EX Lup, combined with the hotter plasma temperature of this component, points clearly to magnetic processes and probably coronal activity as the source of these X-rays. For comparison, the fractional X-ray activity ${ }^{11}$ of EX Lup, during the low-level period, $\log \left(L_{\mathrm{X}} / L_{\mathrm{bol}}\right)=-3.8$, is similar to the median value observed for (accreting) T Tauri stars of the Orion nebula cluster (i.e., -3.74; Preibisch et al. 2005). Moreover, the flare amplitude is well within the range of values observed in this sample for $\mathrm{T}$ Tauri stars having similar value of low-level X-ray luminosity (see Fig. 3b of Stassun et al. 2007).

The large photoelectric absorption of the hotter plasma component of EX Lup is equivalent to an optical extinction of 15 mag (Vuong et al. 2003). However, the value of the optical extinction of EX Lup, although not well known, is very likely much lower, and generally assumed to be 0 or 1 mag in the literature (e.g., Herbig et al. 2001; Gras-Velázquez \& Ray 2005; Sipos et al. 2009). Herbig et al. (2001) noticed that the near-IR colors of EX Lup obtained in March 1992 (Hughes et al. 1994) are redder than those of normal M0 dwarfs. We found here that the reddened color of EX Lup in the near-IR is consistent with the intrinsic near-IR excesses of classical T Tauri stars (CTTSs), which are caused by direct emission of near-IR flux from disks and not by the reddening of photospheric emission by dust. In particular, 2MASS near-IR colors of EX Lup $(J-H=0.770 \pm 0.032 \mathrm{mag}, H-K=0.462 \pm 0.030 \mathrm{mag})$ - obtained on May 16, 1999, when EX Lup was at its minimum level (a visual magnitude of 13.5; Herbig 2007) - are well located on the loci of CTTSs (Meyer et al. 1997) tranformed to the 2MASS color system (using the color transformations of Carpenter 2001). Therefore, we conclude that the optical extinction of EX Lup is fully consistent with the argument that EX Lup experiences no optical extinction $\left(A_{V}=0 \mathrm{mag}\right)$ by dusty material. Moreover, Sipos et al. (2009) argued, from modeling of the spectral energy distribution of EX Lup during quiescence, that the accretion disk is viewed more nearly face-on than edgeon, with a best-fit model inclination of $20^{\circ}$. This low inclination prevents any absorption of the stellar coronal emission by circumstellar material (gas+dust), which is consistent with the null optical extinction.

The large column density is hence necessarily produced by a dust-free gas with a low ionization. The accreted material close to a few stellar radii of the stellar photosphere is naturally dustfree after the sublimation of the dust grains. Moreover, the accretion funnels, loaded with high density gas $\left(\sim 10^{12} \mathrm{~cm}^{-3}\right)$, can easily produce this large column density on a length scale of only 0.3 stellar radii. The more or less pole-on view may also favor the absorption by these accretion funnels of X-ray emission from stellar magnetic loops at lower latitudes (Gregory et al. 2007).

On the surface, the sustained presence of a luminous, hard (coronal) X-ray component toward the end of the outburst of EX Lup would seem to be at odds with models in which energetic ions emitted during coronal flares transform disk dust

\footnotetext{
11 Following Kenyon \& Hartmann (1995), we compute the stellar luminosity of EX Lup, $L_{\mathrm{bol}}$, from its magnitude in the $J$-band - specifically 2MASS (low-level) measurement of $9.73 \mathrm{mag}$, transformed to the homogenized system of Bessell \& Brett (1988) using the color transformations of Carpenter (2001) - using $V-J$ color and bolometric correction appropriate for a M0 spectral type star (see Table A.5 of Kenyon \& Hartmann 1995). We obtain $L_{\mathrm{bol}}=0.55 L_{\odot}$, and a corresponding stellar radius of $1.7 R_{\odot}$ for an effective temperature of $3850 \mathrm{~K}$ (Kenyon \& Hartmann 1995).
} 
grains from silicate to amorphous form (Glauser et al. 2009), given that the reverse was observed during the EX Lup outburst (Ábrahám et al. 2009). We speculate that the large column density observed in front of a coronal active region during the 2008 extreme outburst of EX Lup protected the fresh crystalline silicate apparently produced, against amorphization, hence possibly encouraging the production of cometary material during accretion outburst. Further modeling of the potential effects of PMS outbursts and flares on disk dust-grain structure is clearly warranted.

\subsection{The origin of the soft X-ray component of EX Lup}

The low plasma temperature of the soft component is consistent with X-ray emission from a shock, located either inside a jet, as proposed for TAX sources (Kastner et al. 2005; Güdel et al. 2005; Güdel et al. 2007b), or at the base of an accretion shock, as suggested by analyses of high-resolution X-ray spectra of CTTSs (Kastner et al. 2002; Stelzer \& Schmitt 2004; Schmitt et al. 2005; Günther et al. 2006; Argiroffi et al. 2007; Robrade \& Schmitt 2007; Brickhouse et al. 2010).

The former is unlikely as there is no indication of a jet in EX Lup. Forbidden emission lines of [O I], [N II], and [S II], which are the jet signatures visible in optical spectrum of CTTSs (e.g., Hirth et al. 1997), are not detected in the optical spectra of EX Lup (e.g., Kóspál et al. 2008, and references therein; see in particular Herbig 2007 for Keck/HIRES optical spectrum showing a lack of [S II] emission lines).

Furthermore, in contrast to TAX sources (e.g., Fig. 2 of Güdel et al. 2007b), variability is evident in the soft component of the EX Lup X-ray spectrum. Hence, we favor the latter interpretation of the soft X-ray component, i.e., that this component has its origin in accretion shocks. As in the case of TW Hya (Kastner et al. 2002; Brickhouse et al. 2010), our pole-on view of the EX Lup star-disk system indeed probably provides a direct view of the relatively cool plasma associated with these shocks.

\subsection{The origin of the UV emission of EX Lup}

The UV light curve of EX Lup is reminiscent of the UV activity already observed with the OM from other accreting pre-main sequence stars (e.g., BP Tau; Schmitt et al. 2005). In these accreting systems, the UV emission is directly related to a hot spot on the stellar surface (Calvet \& Gullbring 1998; Gullbring et al. 1998). In this framework of magnetospheric accretion (see review of Bouvier et al. 2007), hot spots are located at the base of magnetic loops, which connect the photosphere and the accretion disk and are loaded with free-falling material that produces accretion shocks at the stellar surface.

Following Schmitt et al. (2005), we estimated the surface filling factor of the hot spots, i.e., the ratio of the hot spot area to the surface of the EX Lup photosphere. Using the UVM2 filter count rate to flux conversion factor ${ }^{12}$ of $2.19 \times 10^{-15} \mathrm{erg} \mathrm{cm}^{-2} \mathrm{~s}^{-1} \AA^{-1}$, we converted the minimum count rate of $\sim 36$ counts $^{-1}$ to $1.8 \times 10^{-10} \mathrm{erg} \mathrm{cm}^{2} \mathrm{~s}^{-1}$. As we discussed in Sect. 4.2, the optical extinction is negligible in EX Lup, therefore, we could consider this value to be the intrinsic UV flux. We assumed for the hot spot a black-body spectrum of temperature $13000 \mathrm{~K}$, as measured from an IUE spectrum obtained during the 1994 outburst, when EX Lup was at $V=12.1 \mathrm{mag}$ (Herbig et al. 2001), i.e., 3.3 times fainter than during our observation. We found a surface filling factor of $0.9 \%$ for the hot spot. This small surface-filling

12 See the OM calibration status (issue 5.0). factor is typical of values found in CTTSs (Calvet \& Gullbring 1998).

The increase by about $45 \%$ in the count rate from the minimum to the maximum observed value over a period of about $10 \mathrm{~h}$ can be explained either by an increase in the surface filling factor of the hot spot in the same proportion, or by an increase of $1000 \mathrm{~K}$ in the hot spot temperature. The latter can be produced by an increase in the mass accretion rate of a factor of $\sim 1.2$ (an estimate we obtained by interpolating the grid of models of Calvet \& Gullbring 1998).

\section{Conclusions}

We observed EX Lup with XMM-Newton a few days before the end of its 2008 extreme optical outburst, but when EX Lup was still in an elevated state. This observation is the longest continuous exposure that was obtained in X-rays and UV during this optical outburst.

We have found that the X-ray light curve of EX Lup contains mainly a low-level period and a two-hour flare event, during which the observed flux increased by a factor of four and noticeably hardened. The variability observed in the UV on timescales ranging from less than one hour up to about four hours can be modeled with a red-noise spectrum. None of the UV events observed on August 10-11, 2008 correlate unambiguously with simultaneous X-ray peaks. This UV activity is typical of accretion events and is dominated by emission from accretion hot spots covering only about one percent of the stellar surface.

The X-ray spectrum of EX Lup reveals two plasma components, one that is cooler with low absorption and one that is hotter with high absorption. The intrinsic X-ray luminosity of EX Lup is dominated by the emission from the hot plasma component. During the X-ray flare, the emission measure and the intrinsic X-ray luminosity of this absorbed plasma component is five times greater.

The soft plasma component is most likely associated with X-ray emission from accretion shocks - an inference based mainly on the absence of a jet in EX Lup. The nearly pole-on viewing geometry likely explains the high absorption of X-ray emission from low-latitude active regions by the high-density accretion funnel flows. Hence, the same dust-free accreting material that generates the soft component (via shocks) also largely smothers the corona of EX Lup. This model predicts that large values of column density, such as we measured toward the hard spectral component of EX Lup near the end of its 2008 outburst, should be present only during these accretion-driven optical outbursts.

Acknowledgements. We thanks the XMM-Newton Science Operations Centre for the prompt schedule of this observation. This research is based on observations obtained with XMM-Newton, an ESA science mission with instruments and contributions directly funded by ESA Member States and NASA. We acknowledge with thanks the variable star observations from the AAVSO International Database contributed by observers worldwide and used in this research. J.K.'s research on X-rays from erupting YSOs is supported by NASA/GSFC XMM-Newton Guest Observer grant NNX09AC11G to RIT.

\section{Appendix A: Correcting OM fast-mode light curve of spurious features caused by spacecraft drifts and missing $\mathrm{OM}$ tracking history file}

Before each OM science observation, a short-exposure image, called a Field AcQuisition (FAQ), is taken with the $V$-filter to identify proper field stars by comparison with the uploaded guide-star catalog. These good guide stars are then used as a 
N. Grosso et al.: accretion shocks and a smothered stellar corona unveiled by XMM-Newton

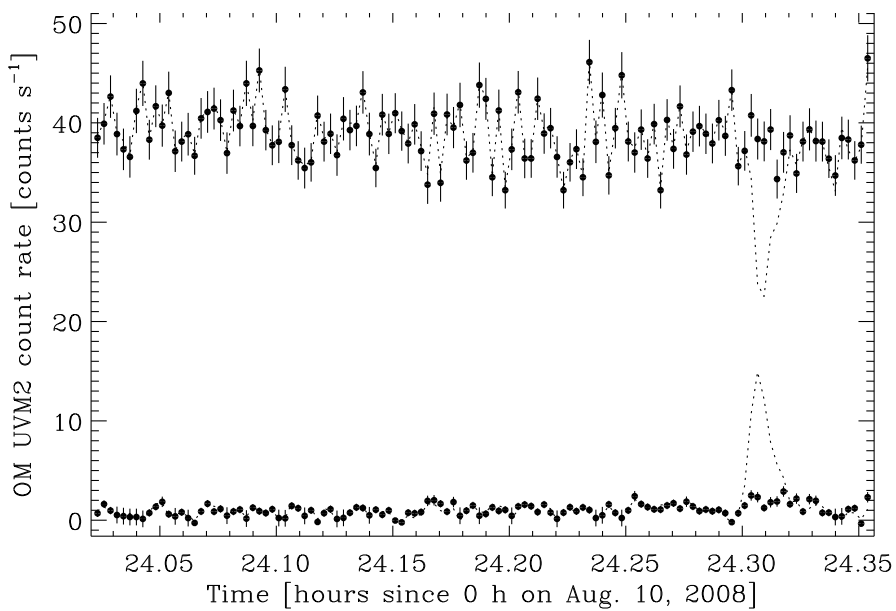

Fig. A.1. UV light curves for EX Lup (top, with the background subtracted) and for the background (bottom) during the S415 exposure of the $\mathrm{OM}$ in the fast mode. The dotted lines show the light curves obtained with the SAS script omfchain and no tracking history file. The data points show the corrected light curves using our spacecraft drift estimates. The interval of the time bin is $10 \mathrm{~s}$.

reference frame to center the fast-mode window (composed of $22 \times 23$ pixels, each pixel having a size of $\sim 0$ '. 48 , i.e., covering a field-of-view of about $\left.10.5 \times 11^{\prime \prime}\right)$ on the target at the start of the science exposure, and to monitor any spacecraft drifts during the OM science observation. However, owing to telemetry constraints, no shift-and-adds of the fast-mode frames are performed on the spacecraft. Corrections of spacecraft drifts are made on the ground using the OM tracking history file (*OM*THX.FIT). When no good guide stars are found during the FAQ, the target cannot be centered on the fast-mode window, and any spacecraft drift during the exposure moves the target on the detector, which produces flux losses when aperture photometry is performed.

During our observation of EX Lup with the OM fast mode, no good guide stars were found during the FAQ; therefore, the OM tracking history file was not written to the Observation Data Files. For illustration purposes, we focused here on the S415 exposure, although several other exposures were found to exhibit similar artifacts.

Figure A.1 shows the light curves for EX Lup and the background, obtained using a 6-pixel extraction radius around EX Lup and an extraction radius of 1.2 to 2.5 times the source extraction radius for the background. These light curves were obtained with the SAS script omfchain, which assumes here zero spacecraft drifts (see dotted lines in Fig. A.1). The light curves for EX Lup and the background exhibit a dip and a peak, respectively, at about $24.31 \mathrm{~h}$, lasting about 1 minute, and have identical profiles. Therefore, these features are not real, but artifacts that can be explained by a decentering of the target with respect to the apertures, i.e., a motion of the target on the detector caused by spacecraft drifts.

To estimate the spacecraft drifts, we propose using the OM fast-mode event list file (*OM*FAE.FIT) that provides for each event its frame number and its detector position (RAWX and RAWY coordinates). We track any motion of the target on the detector versus time by calculating the difference between the moving median and the median value of both detector coordinates versus times, with our IDL program. For the moving median, we use a set of frames containing at least 500 photons, which corresponds for EX Lup to a centered time interval with a half-width of about 6 s. The top and bottom panels of Fig. A.2 show the scattered
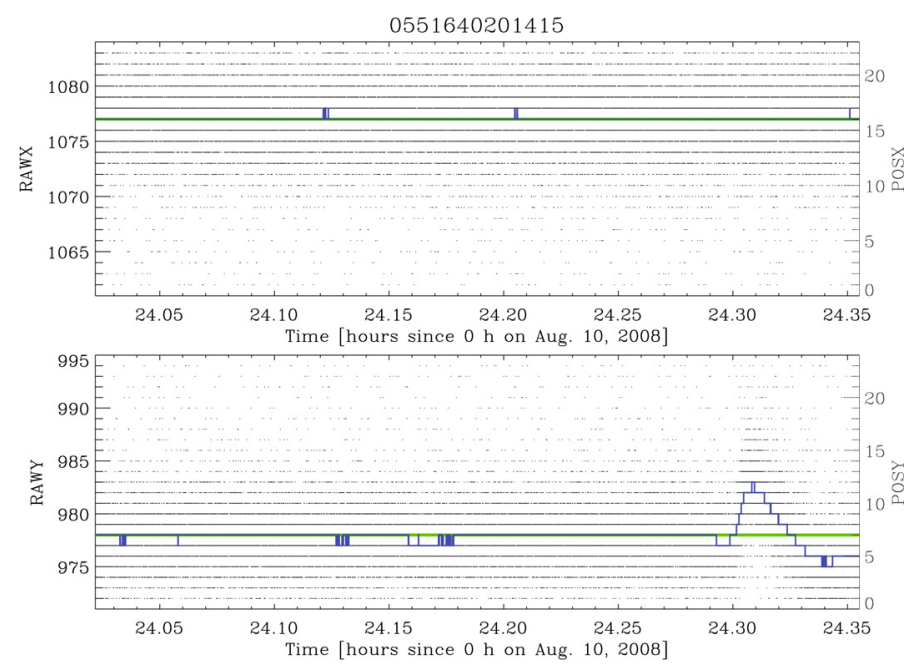

Fig. A.2. OM fast-mode window positions versus arrival times of UV photons detected during the S415 exposure. The top and bottom panel are the scattered plot of the positions of the UV photons detected by the optical monitor on the $x$ and $y$-axis of the fast-mode window, respectively, versus the frame times corresponding to the photon arrival times. The left and right axis give the detector and the fast window coordinates, respectively. The green line show the median position during the exposure. The blue line shows the moving median position versus time computed on a set of frames containing at least 500 photons. (This figure is available in color in electronic form.)

plots of RAWX and RAWY, respectively, versus time. The green and blue lines are the median position during the exposure and the moving median position versus time, respectively. A drift of 5 pixels along the detector $y$-axis of the target (corresponding to a motion of $\sim 2$.' 5 on the sky), which occurred simultaneously with the features of Fig. A.1, is visible.

To correct the OM fast-mode event list of these spacecraft drifts, we wrote a bash script, using SAS and ftools tasks, based on the SAS thread entitled "OM data reduction with SAS: step-by-step fast mode data processing chain" "13. This SAS threads lists the 14 steps that are needed to process the fast-mode data with SAS tasks, and describes intermediate output files. In particular, the SAS task omfastshift (step 6) adds to the event list the new columns CORR_X and CORR_Y, but using here zero drifts (i.e., CORR_X=RAWX and CORR_Y=RAWY). Therefore, immediately after this step, we introduce ftools commands to add to these columns our estimate of the spacecraft drifts. From these corrected positions, we produce a corrected image in the detector frame from which the source detection is performed (step 8). Figure A.1 shows OM fast-mode light curves obtained with this method. The spurious features due to spacecraft drifts have been corrected.

We note that the SAS task omlcbuild (step 13) restores the fraction of point spread function (PSF), which falls out of the fast-mode window on the final corrected image, and not on the individual frames. Therefore, for frames where the spacecraft drifts are so large that the target is close to the window edges, the fraction of PSF which falls out of the window cannot be totally restored. For these frames, the spurious features will be only mitigated. This limitation exists even when the OM tracking history file is not missing.

13 This SAS thread is available at:

http://xmm.esac.esa.int/sas/current/documentation/ threads/omf_stepbystep.shtml. 


\section{References}

Ábrahám, P., Juhász, A., Dullemond, C. P., et al. 2009, Nature, 459, 224 Anders, E., \& Ebihara, M. 1982, Geochim. Cosmochim. Acta, 46, 2363 Anders, E., \& Grevesse, N. 1989, Geochim. Cosmochim. Acta, 53, 197 Argiroffi, C., Maggio, A., \& Peres, G. 2007, A\&A, 465, L5 Audard, M., Güdel, M., Skinner, S. L., et al. 2005, ApJ, 635, L81

Audard, M., Stringfellow, G. S., Güdel, M., et al. 2010, A\&A, 511, A63 Bessell, M. S., \& Brett, J. M. 1988, PASP, 100, 1134

Bouvier, J., Alencar, S. H. P., Harries, T. J., Johns-Krull, C. M., \& Romanova, M. M. 2007, in Protostars and Planets V, ed. B. Reipurth, D. Jewitt, \& K. Keil, 479

Brickhouse, N. S., Cranmer, S. R., Dupree, A. K., Luna, G. J. M., \& Wolk, S. 2010, ApJ, 710, 1835

Calvet, N., \& Gullbring, E. 1998, ApJ, 509, 802

Carpenter, J. M. 2001, AJ, 121, 2851

Comerón, F. 2008, The Lupus Clouds, in Handbook of Star Forming Regions, Volume II: The Southern Sky, ed. B. Reipurth, ASP Monograph Publ., 5, 295

Dorman, B., \& Arnaud, K. A. 2001, in Astronomical Data Analysis Software and Systems X, ed. H. E. P. F. R. Harnden Jr., \& F. A. Primini, ASP Conf. Ser., 238, 415

Feigelson, E. D., \& Montmerle, T. 1999, ARA\&A, 37, 363

Getman, K. V., Flaccomio, E., Broos, P. S., et al. 2005, ApJS, 160, 319

Glauser, A. M., Güdel, M., Watson, D. M., et al. 2009, A\&A, 508, 247

Gras-Velázquez, À., \& Ray, T. P. 2005, A\&A, 443, 541

Gregory, S. G., Wood, K., \& Jardine, M. 2007, MNRAS, 379, L35

Grosso, N. 2006, in The X-ray Universe 2005, ed. A. Wilson, ESA SP, 604, 51

Grosso, N., Kastner, J. H., Ozawa, H., et al. 2005, A\&A, 438, 159

Güdel, M., \& Nazé, Y. 2009, A\&ARv, 17, 309

Güdel, M., Skinner, S. L., Briggs, K. R., et al. 2005, ApJ, 626, L53

Güdel, M., Briggs, K. R., Arzner, K., et al. 2007a, A\&A, 468, 353

Güdel, M., Telleschi, A., Audard, M., et al. 2007b, A\&A, 468, 515

Gullbring, E., Hartmann, L., Briceño, C., \& Calvet, N. 1998, ApJ, 492, 323

Günther, H. M., Liefke, C., Schmitt, J. H. M. M., Robrade, J., \& Ness, J.-U. 2006, A\&A, 459, L29

Hamaguchi, K., Grosso, N., Kastner, J. H., Weintraub, D. A., \& Richmond, M. 2010, ApJ, 714, L16

Hartmann, L., \& Kenyon, S. J. 1996, ARA\&A, 34, 207

Herbig, G. H. 1966, Vistas in Astron., 8, 109

Herbig, G. H. 1977a, ApJ, 217, 693

Herbig, G. H. 1977b, ApJ, 214, 747

Herbig, G. H. 1989, in ESO Workshop on Low Mass Star Formation and PreMain Sequence Objects, ed. B. Reipurth, European Southern Observatory Astrophysics Symp., 33, 233

Herbig, G. H. 2007, AJ, 133, 2679

Herbig, G. H. 2008, AJ, 135, 637
Herbig, G. H., Aspin, C., Gilmore, A. C., Imhoff, C. L., \& Jones, A. F. 2001, PASP, 113, 1547

Hirth, G. A., Mundt, R., \& Solf, J. 1997, A\&AS, 126, 437

Hughes, J., Hartigan, P., Krautter, J., \& Kelemen, J. 1994, AJ, 108, 1071 Itagaki, K., Nakano, S., \& Yamaoka, H. 2008, IAU Circ., 8968, 2

Jones, A. F. 2008a, Central Bureau Electronic Telegrams, 1217, 1

Jones, A. F. 2008b, Central Bureau Electronic Telegrams, 1231, 1

Kastner, J. H., Huenemoerder, D. P., Schulz, N. S., Canizares, C. R., \& Weintraub, D. A. 2002, ApJ, 567, 434

Kastner, J. H., Richmond, M., Grosso, N., et al. 2004, Nature, 430, 429

Kastner, J. H., Franz, G., Grosso, N., et al. 2005, ApJS, 160, 511

Kastner, J., Richmond, M., Simon, T., et al. 2006a, Central Bureau Electronic Telegrams, 760, 1

Kastner, J. H., Richmond, M., Grosso, N., et al. 2006b, ApJ, 648, L43

Kenyon, S. J. \& Hartmann, L. 1995, ApJS, 101, 117

Kóspál, A., Nemeth, P., Abraham, P., et al. 2008, Inform. Bull. Variable Stars, 5819,1

Lomb, N. R. 1976, Ap\&SS, 39, 447

Lombardi, M., Lada, C. J., \& Alves, J. 2008, A\&A, 480, 785

Lorenzetti, D., Giannini, T., Calzoletti, L., et al. 2006, A\&A, 453, 579

Mason, K. O., Breeveld, A., Much, R., et al. 2001, A\&A, 365, L36

Meyer, M. R., Calvet, N., \& Hillenbrand, L. A. 1997, AJ, 114, 288

Morrison, R., \& McCammon, D. 1983, ApJ, 270, 119

Pojmanski, G. 2002, Acta Astron., 52, 397

Preibisch, T., Kim, Y.-C., Favata, F., et al. 2005, ApJS, 160, 401

Press, W. H. 1978, Comm. Astrophys., 7, 103

Press, W. H., \& Rybicki, G. B. 1989, ApJ, 338, 277

Robrade, J., \& Schmitt, J. H. M. M. 2007, A\&A, 473, 229

Scargle, J. D. 1982, ApJ, 263, 835

Schmitt, J. H. M. M., Robrade, J., Ness, J.-U., Favata, F., \& Stelzer, B. 2005, A\&A, 432, L35

Sipos, N., Ábrahám, P., Acosta-Pulido, J., et al. 2009, A\&A, 507, 881

Skinner, S. L., Briggs, K. R., \& Güdel, M. 2006, ApJ, 643, 995

Skinner, S. L., Sokal, K. R., Güdel, M., \& Briggs, K. R. 2009, ApJ, 696, 766

Stassun, K. G., van den Berg, M., \& Feigelson, E. 2007, ApJ, 660, 704

Stecklum, B. 2006, Central Bureau Electronic Telegrams, 690, 1

Stecklum, B., Melnikov, S. Y., \& Meusinger, H. 2007, A\&A, 463, 621

Stelzer, B., \& Schmitt, J. H. M. M. 2004, A\&A, 418, 687

Stelzer, B., Hubrig, S., Orlando, S., et al. 2009, A\&A, 499, 529

Strüder, L., Briel, U., Dennerl, K., et al. 2001, A\&A, 365, L18

Szeifert, T., Hubrig, S., Schöller, M., et al. 2010, A\&A, 509, L7

Timmer, J., \& Koenig, M. 1995, A\&A, 300, 707

Turner, M. J. L., Abbey, A., Arnaud, M., et al. 2001, A\&A, 365, L27

Uttley, P., McHardy, I. M., \& Papadakis, I. E. 2002, MNRAS, 332, 23

Vuong, M. H., Montmerle, T., Grosso, N., et al. 2003, A\&A, 408, 581 